solution of permanganate of potassium is useful, and he still practices that procedure, but it is not absolutely preventive. In his opinion the prophylactic power of the bichlorid ointment is due to its remaining in the lachrymal canals, partially plugging them, and thus presenting a mechanical as well as chemical barrier to the entrance of germs from the nasal passages.

DR. E. E. Holt, Portland, Maine, stated that he has had one suppuration in 200 cases. He is still, however, very much in favor of the method and uses it in every case.

DR. C. R. Holmes, Cincinnati, said that it was his misfortune, early in his career, to lose a case from an infected tear sac, and that led to his following the method of extirpating the sac and gland. He would refuse to operate where there is a purulent inflammation in the tear sac unless he could extirpate the sac. He has performed this operation in a patient over 80 years old with perfect success. He long ago abandoned the idea of trying to elean the mucous membrane that has not shown any inflammation prior to the operation, as he thinks that this only sets up an irritation that gives a good chance for infection. He does not use anything at all except sterile water, or a saturated solution of boracic acid.

Dr. V. T. Churchman, Charleston, W. Va., said that since Dr. White spoke to him several years ago about this method of preparing sterilized sublimated vaselin, he has used it in every case on which he has operated since. He had infection in only one case and that subsided promptly on following up the treatment.

Dr. J. L. Borscrr, Philadelphia, said that one point not brought out is the cleansing and sterilization of the lashes prior to operation. He does not believe it is the proper thing to use very strong solutions in sterilizing the conjunctival sac; they seem to make infection more probable. De Wecker simply cleanses and sterilizes the lids and lashes with cyanid of mercury, 1 to 100 , then flushes the conjunctival sac with a boracic acid solution and follows this with a douche of sterile distilled water. Dr. Borsch follows this method and finds it entirely satisfactory.

Dr. F. P. Lewis, Buffalo, N. Y., said that he has had in two cases a suspicion of infection, one from the sac and the other from without. He has wondered, in view of the success of the Crede method, that the silver salts have not been more used in these cases. With that idea in mind some years ago he made it a routine practice, in attempting to thoroughly cleanse the eye, to use a solution of 2 or 3 per cent. protargol in the eye just prior to operation. It is non-irritating and Crede's experiments have shown that there is certainly a lessened probability of infection in gonorrheal ophthalmia. It would seem reasonable, therefore, to suppose that like protective results might be expected in operative cases. Since using it he has had no infections.

Dr. J. A. White said that the actual treatment of the infection itself and how to get rid of it is just as much in the dark as it was before. Each one has his own methods for the prevention of infection. He thinks that in his case the infection came from within, perhaps because he did not investigate the condition of the nose properly before operation. He included cleansing the lashes in the prior cleansing of the eyelids as given in the paper, viz., scrub with J to 1,000 bichlorid, and then wash with alcohol before applying the ointment to the eye and lids.

Honest Experts Wanted.-Dr. S. Solis-Cohen read a very interesting paper at the pharmaceutical meeting in the Philadelphia College of Pharmacy, November 15, on "The True Scope of Scientific or So-Called Expert Testimony in Trials Involving Pharmacological Questions." He said that the expert should distort, magnify or minimize nothing, assert as positive nothing doubtful, throw doubt on nothing certain, and say nothing he would not defend before a learned society. He further said the expert's efforts should be bent to elucidate truth and not to score points for either side. The court should restrict examination to matters having actual bearing on the case at trial; but the testimony of the expert should be given only with reference 10 scientific accuracy, and not with reference to its effect on the verdict.

\section{SEPTIC THROMBOSIS OF THE CAVERNOUS SINUSES.}

WITH A REPORT OF THREE CASES.*

\section{E. C. ELLETT, M.D. \\ MEMPHIS, TENN.}

CASE 1.-J. W., white, male, age 15, of good family history and previously healthy; was taken sick Aug. 2, 1898, with pain in and around the right eye. This pain was severe, but I was not able to ascertain if it was accompanied by other local symptoms, or if there was fever. The pain was relieved by acetanilid, but returned next day, and there was then swelling over the brow and fever.

Examination.-These symptoms persisted, and he was brought to Memphis on the fifth day of his illness and was placed in the care of a general practitioner. There was a fluctuating swelling over the frontal region, extending up to the hair and down to the root of the nose. This was incised at its upper border and pus escaped. On the next day an incision was made above the root of the nose for better drainage. On the ninth day of his illness I was asked to see him on account of an involvement of the eyes, especially the left. $I$ found the two openings as described, discharging pus from a common cavity. The eyelids were swollen till the eyes could not be voluntarily opened. A fluctuating swelling was seen at the upper and inner corner of the right orbit, and the left eye showed exophthalmos, with fixation of the ball, edema of the conjunctiva and ulceration of the cornea at its lower margin. The pupils were normal. The skin was of a pale yellow, temperature 103 to 104 degrees, and a small abscess was found over the left parietal region. The pulse was rapid, mind clear. In other words, there was general septic infection, apparently having its origin in the right frontal sinus.

Operation.-Under general anesthesia the abscesses were freely opened, the left frontal sinus opened and found full of pus, the lining thickened, and an opening in the septum between it and the right sinus. The sinuses were cleaned out, thrown into one, and opened freely into the left nostril. Pus was present in the right orbit, none in the left. The operation was done at $11 \mathrm{a} . \mathrm{m}$. and the collections of pus being liberated and provision made for drainage, a hopeful view was taken of the case. We did not recognize the fact that we had to deal with anything more than the accessible foci of suppuration. At $2 \mathrm{p} . \mathrm{m}$. the temperature was 105 degrees, and briefly, all the symptoms grew worse. A general surgeon was called in consultation and could suggest nothing further than repeated exploration of the left orbit for pus, which was never found. Stupor developed and on the fourteenth day of his illness a right hemiplegia appeared. The patient was then comatose, and without further ehange in the local symptoms except a spread of the corneal ulceration in the left eye, he died on the fifteenth day of his illness. No autopsy was performed.

CASE 2.-N. G., white, male, aged 27 , was seen in consultation with Dr. W. T. Michie, through whose kindness I am permitted to report the case, on June 20, 1903. Six days previously he had consulted Dr. Michie for a phlegmonous inflammation of the right ala nasi of two days' duration. It had started as the common furuncle in this situation usually does, but was, when seen by Dr. Michie, so severe and so painful that a general anesthetic was given and a free incision made. On the following day the nose was red and swollen to the root, and both eyelids somewhat swollen. On the next day the symptoms were all worse, with elevation of temperature, swelling of the right eyelids, nose, forehead and right side of the face and neck. In the mouth a right peritonsillar swelling was seen. All the swellings were edematous rather than inflammatory.

Examination.-When I saw him on the eighth day of his illness there was a swelling and redness of the nose and right lid, and slight swelling at the inner angle of the left orbit.

- Read at the Fifty-fifth Annual Session of the American Medical Association, in the Section on Ophthalmology, and approved for publication by the Executive Committee: Drs. Frank Allport John E. Weeks and R. L. Randolph. 
The peritonsillar swelling persisted, as did the edema of the right side of the face and neck. There was right exophthalmos, the orbit being tense, and conjunctiva edematous (right), but the ball itself was normal, the pupil reacting and motion being unimpaired. Temperature 102.5. He had had several chills. I gave my opinion that a septic thrombus existed in the right cavernous sinus, just beginning to involve the left one, and that the patient would die. I saw him again on the eleventh day of his illness. He was then unconscious, but could be roused to answer questions. Incisions into the peri. tonsillar swelling had evacuated a quantity of dark blood, but no pus. The temperature was running from 100 to $102.5 \mathrm{de}-$ grees, the pulse was rapid and weak, respiration 50 . He was restless, tossing from side to side and pulling at the bedclothes. No signs of consolidation in the lungs could be made out, though there must have been one or more metastatic abscesses. There was double exophthalmos. On the right side the conjunctiva was hemorrhagic, slight dilatation of the pupil, and divergent squint (oculomotor paralysis). The left eye was normal except for edema of the lids and conjunctiva, and a black, dry slough in the center of the upper lid the size of a dime. A similar large area was seen on the bridge of the nose and a large oval bluish spot on the forehead. The edema of the right side of the face and neck was worse. The patient died at 7 o'clock that evening. No autopsy.

CASE 3.-G. W., white, male, aged 27, consulted my associate, Dr. Farrington, on Oct. 2, 1903, with a small pustule at the end of the right nostril. This was gently ruptured, swabbed with bichlorid of mereury and a wash of the same solution given for use at home. The next day the parts were still inflamed and the process was repeated. On the 4 th he was seen by his family physician, Dr. Malone, who was at the house to see our patient's child. The nose was still red, swollen and painful, and learning what had been done, Dr. Malone did not give any active treatment, but on the following day, October 5 , the symptoms not abating, Dr. Malone made a free incision of the inflamed area under local anesthesia. There was no evidence at this time of any extension beyond the nose, but on the 6th the morning temperature was 102 degrees and the lid of the right eye was swollen. That evening the temperature was 104 degrees and the pain was intense. On the morning of the 7th Dr. Farrington saw him with Dr. Malone and made the diagnosis of septic thrombosis of the right cavernous sinus.

Examination.-The patient was removed to our infirmary, where I saw him at 2 p. m. on this, the fifth day of his illness. The nose was red and swollen, and there were several small superficial pustules at the end. The lid of the right eye and the skin of the forehead were edematous, a phlebitis of the supraorbital veins could easily be made out, the conjunctiva of the right eye was chemotic and swollen and the ball fixed, pupil slightly dilated and vision obscured as by a white nist before the eye. The lids of the left eye were slightly swollen at the inner corner. Temperature 104 degrees, pulse 112 , mind clear.

operation.-Under chloroform the nose was freely incised and some pus escaped. The right orbit was incised, but no pus was found. Six drams of antistreptococcic serum was administered, but had no effect and was not repeated. The treatment was symptomatic, and no material change could be suggested by any of several consultants, among them Dr. A. J. Ochsner of Chicago, who was in the city attending a meeting of the Mississippi Valley Medical Association. On the sixth day the conjunctiva of the left eye became edematous, the temperature remaining high and the pulse rapid. That night the patient began to sink rapidly and became delirious, and next morning (seventh day) was weaker, with temperature 105 degrees, cold extremities and active delirium. The right eye was divergent with dilated pupil. Death at noon of the seventh dav. No autopsy.

It is a question whether this is the proper place to report cases of this condition, but $I$ do so because these caces were scen by me on account of apparent eve symptoms. Moreover, the disease is rare, and as this rather large number of eases have fallen under my notice, I venture to relate them for the purpose of calling attention to the condition.

The literature on this subject is not abundant by any means. Dwight and Germain ${ }^{1}$ append an extensive bibliography to their report of 4 cases, and were able to find 178 cases, with their 4, making 182. I would refer those interested in the literature to this article. 'The importance of the condition lies in its great mortality, only 14 of these 182 cases recovering. In septic cases the prognosis is always bad. Since this collection of cases others have been reported by Day, ${ }^{2}$ Lodge ${ }^{3}$ and Finlay. ${ }^{4}$

Day's patient was a child of 11 , suffering with chronic purulent otitis media and mastoiditis, both of the right side. Five days after operation the right eye was swollen, and thirteen days later the left eye was similarly involved. Fluctuations in the temperature seemed to be due to retention of pus in the mastoid wound, and though both cornea ulcerated the child recovered, leaving the hospital at the end of four and onehalf months. Largely on account of recovery, Day thinks the thrombi in the cavernous sinuses must have been non-infective and ultimately absorbed. Its association with mastoid disease he regards as a coincidence. The lateral sinus was not involved, though cases have been observed where the infectious process spread from this sinus to the cavernous by way of the superior petrosal.

Lodge's case was a married woman, aged 41. The trouble originated in the left peritonsillar region, spreading to the cavernous sinus of the left side by way of the pterygoid plexus. Pus was found on incision back of the last molar tooth, and later a purulent discharge came from the left nostril. The antrum of Highmore was healthy. Autopsy verified the diagnosis, there being septic thrombi in both cavernous sinuses and pus distributed pretty well over the base. The sphenoidal sinus was full of pus, other accessory sinuses healthy. He thinks there was a primary marasmic thrombus of the pterygoid plexus which became infected from the mouth.

In Finlay's case all the symptoms pointed to mastoid and lateral sinus disease, complicating acute otitis media. On the operating table a swelling under the left superior orbital margin led the operator to diagnose a complicating thrombosis of the cavernous sinus. The lateral sinus was found healthy and the mastoid practically so. Autopsy showed a purulent clot of the cavernous and circular sinuses, extending to the left ophthalmic vein, and pus in the sphenoidal and posterior ethmoidal cells. No symptoms of nasal disease had ever been complained of. Finlay remarks that "one can scarcely seriously contemplate reaching the local lesion through the orbit or by means of a craniectomy."

By far the most lucid and satisfactory exposition of the subject that I have seen is in McEwen's "Pyogenic Diseases of the Brain and Spinal Cord," and what can be said on the subject in general is excellently said by him.

The cause of this condition. i. e., septic thrombosis of the cavernous sinus, is any infected lesion in the area drained by the ophthalmic vein or its branches. Pustules on the face. nostrils, evelids. etc., purulent affections of the accessory sinuses, nasal, pharyngeal or

1. Baston Med. and Surg. Iour.. Mar 1, 1902.

2. Annals of Otologv. vol. xi. p. 520 .

3. Annals of Otolory. vol. xil. p. 449.

4. Archlves of $n+0 \log y, \nabla o l, x x x i 1$, p. 419 
buccal cavities, facial erysipelas, infected wounds of this region, etc., may be the cause. Here we encounter the first important point, which is the necessity of care and cleanliness in dealing with pustules or other infected lesions of the face. Two of my three cases resulted from lesions which the rhinologist sees almost daily and which most of us have personally experienced.

The symptoms naturally divide themselves into (1) the local and (2) the general. The general symptoms are those of sepsis under any and all circumstances. The local symptoms may, following McEwen's scheme, be divided into $(a)$ those due to venous obstruction, and (b) those due to pressure on the nerves. Venous obstruction causes edema and chemosis of the affected area, viz., the orbit, the skin of the nose, forehead, cheek and sometimes fauces, pharynx and neck. We have seen in Case 2 that necrosis of the skin may ultimately result. The exophthalmos, which is a prominent symptom, is due to engorgement of the tissues of the orbit.

Pressure on the nerves causes characteristic symptoms. The nerves involved are the second, third, fourth, sixth and the ophthalmic division of the fifth. The visual disturbances are usually very marked, due to pressure on the optic nerve and edema of the retina, while the motor disturbances are most pronounced in the parts supplied by the third. We have seen the dilated pupil, divergent squint and ptosis in the cases reported. The ophthalmoscope shows dilated and tortuous retinal veins and edema of the retina. The disturbances due to pressure on the fifth are variable.

A very important and characteristic symptom arises from the fact that while the trouble is unilateral at first, the thrombus soon spreads to the other sinus, and obstruction and other symptoms appear in the areas drained by it. The edema of the lids of the second eye, beginning at the inner canthus, and not due to direct extension, the parts at the root of the nose lying between the eyes being normal, is very characteristic.

The diagnosis must be made from tenonitis, orbital cellulitis and facial erysipelas. If attention is once drawn to the condition under consideration the diagnosis is not difficult, and it is for the purpose of calling your attention to it that $I$ have presented this report.

The prognosis in septic cases is, I believe, uniformly bad. In one of the series reported by Dwight and Germain, operation was performed and they think the results are promising Hartley demonstrated its feasibility in Knapp's well-known case, but the question is probably one for the general surgeon, and no general surgeon who saw any of these cases entertained the idea of operative treatment. Since the prognosis otherwise is absolutely bad, it seems to me proper that operation should be tried. Other than this the treatment is supporting and symptomatic.

Dwight and Germain stand alone in their advocacy of operation, but it is to be hoped that their encouraging results will lear others to give the matter a trial. There is nothing to lose.

\section{DISCUSSION.}

DR. S. D. RISLEY, Philadelphia-I have often been surprised that thrombosis of the cavernous sinus does not occur more frequently. I have a suspicion that it occasionally occurs without being recognized. For many years there has been a recognition of the fact that abscesses about the face were dangerous, probably without recognizing the probability of thrombus of the sinus. A few years ago I saw an aged man who had an abscess of the lachrymal sac on one side. A homeopathic surgeon operated on it. Just what he did I do not know, but in forty-eight hours the patient died with symptoms of thrombosis of the cavernous sinus. I have seen one case of thrombosis. A small abscess occurred at the superior aspect of the malar bone, becoming evident on a Thursday; on Friday there was severe headache, which increased in severity, and on Saturday there was an edema of the left eyelid, with slight proptosis. On Sunday morning there was edema of both lids, the exophthalmos rapidly increased on both sides and the pain became violent and was rendered worse by cold or hot compresses. In this case $200 \mathrm{Swedish}$ leeches declined to draw blood and artificial leeches had to be used. The abscess proved to be of streptococcic infection. Ether was administered and exploration of the orbit was made, but no pus was found there. On Sunday afternoon, the pain being violent, a hypodermic of morphia was given and the patient was immediately relieved from pain and passed into sleep which ended in profound coma that lasted until death the following Wednesday, five days after the onset of the small abscess on the malar bone. This was situated within the area drained by the cavernous sinus. These case suggest the importance of consideration of this subject. When we have an infectious process the area of which drains into the cavernous sinus we should be on our guard for thrombosis of the sinus.

Dr. L. A. Prffontaine, Springfield, Mass.-About six weeks ago I was called to see a young man who three weeks previously suffered from a febrile disease, which was thought at the time to be pneumonia. A week before he had severe pain in the back of the head, in the right thigh and in the right ear. The pain continued and a discharge from the ear began at the end of forty-two hours. Seventy-two hours later I was sent for. He then had a purulent mastoiditis, with a temperature of 103 , pulse 120 , and some delirium, and more severe general illness than would be accounted for the mastoiditis alone. I operaated on the right mastoid and exposed the sinus, which was normal. Five days later there was swelling of both lids and some exophthalmos. Two days later I found him delirious, with temperature 104 , pulse 90 , and marked bilateral exophthalmos; I made the diagnosis of double cavernous sinus thrombosis. Death followed in two days. No postmortem.

Dr. H. S. MrLes, Bridgeport, Conn.-I would add one case, which began in a small abscess in the right nostril. It was opened, the pus examined and found to be or staphylococcus origin. The patient became worse, and on the fourth day was unconscious; the lids were swollen and there was some exophthalmos. No pus was found in the orbit. On the fifth day the patient died.

DR. E. C. ELLETT-My only object in presenting this paper is that these cases are apt to be seen by the ophthalmologist, and should be borne in mind. The diagnosis is easy if attention has been called to the trouble.

\section{PRESBYOPIA.}

\section{E. J. GARDINER, A.B., M.D.}

Professor of Ophthalmology, Chicago Eye, Ear, Nose and Throat College.

CHICAgo.

Not long ago a number of medical men were gathered after luncheon at one of the down-town clubs. They were all about the same age-somewhere between 45 and 50 - and were amusing themselves by gently twitting one of the number who was wearing his first glasses for presbyopia. This twitting led to a discussion, brought about by my defense of the wisdom manifested by the victim in wearing glasses for reading. A test was made in situ with small print, which revealed that every man present was presbyopic. This was not surprising, but the interesting feature to me was, first the aversion they all had to admit the presence of presbyopia, and then the reasons advanced for not using glasses for its correction. No one, of course, wishes to admit a failure of the faculties. That a perfectly normal change, brought about by a maturing 